\title{
NEO search telescope in China
}

\author{
Yuehua $\mathbf{M a}^{1,2}$, Haibin Zhao ${ }^{1,2}$ and Dazhi Yao ${ }^{1,2}$ \\ ${ }^{1}$ Purple Mountain Observatory, Chinese Academy of Sciences, Nanjing 210008, China \\ ${ }^{2}$ National Astronomical Observatories, Chinese Academy of Sciences, Beijing 100012, China \\ email: yhma@pmo.ac.cn
}

\begin{abstract}
Chinese scientists have contributed many research works to the field of asteroid surveying and related topics. In the early 1960s, Purple Mountain Observatory (PMO) began observing asteroids and found over 130 new numbered asteroids during the following decades. The Schmidt CCD Asteroid Program (SCAP) of Beijing Astronomical Observatory (BAO) started in 1995 and found 575 asteroids in several years.

NEOs represent a great threat to the near-Earth environment by closely approaching Earth or even impacting with Earth. After the impact of comet SL9 on Jupiter, Chinese proposed to build a NEO Search Telescope to take part in the joint international NEO survey. It is a 1.0/1.2 $m$ Schmidt telescope with a $4 \mathrm{~K} \times 4 \mathrm{~K}$ drift scanning CCD detector. Here we elaborate upon its applications to objects of scientific interest, its parameters, and its observing station.
\end{abstract}

Keywords. Survey; telescope; observation site

\section{Introduction}

Many Near Earth Object (NEO) Searching Plans are being carried out all over the world, e.g. LINEAR (Stokes, Evans, Viggh, et al. 2000), SPACEWATCH (Gehrels \& Jedicke 1995) etc. China is one of the countries paying great attention to the space environment near Earth. In 1928, Asteroid (1125) China was found at Yerkes Observatory by C.Y. Chang who was the former director of Purple Mountain Observatory (PMO). In 1955, Asteroid Purple 1 was found at PMO by C.Y. Chang \& J.X. Zhang, which was the first asteroid found by Chinese at home. Up to the mid-1980s, the PMO asteroid research group found over 130 new numbered asteroids with a $40 \mathrm{~cm}$ double-tube refracting telescope. Among them, Asteroids (2077) Jiangsu and (2078) Nanjing are Mars-crossing asteroids.

The Schmidt CCD Asteroid Program (SCAP) of Beijing Astronomical Observatory (BAO) was put into practice in 1995 . The telescope used is a 60/90 $\mathrm{cm}$ Schmidt type with a $2 \mathrm{~K} \times 2 \mathrm{~K}$ CCD detector. In 1997, 2 NEOs and a Mars-crossing asteroid were found by SCAP. 575 asteroids were found by this scheme in the 1990s, which performance was among the top in the world at that time.

In 1994, Professor J.X. Zhang and his colleagues predicted the impact of comet Shoemaker-Levy 9 (SL9) on Jupiter. Although lacking the last key observational data, their prediction agreed, to a high degree of accuracy among all those in the world, with the actual one observed by the Galileo spacecraft.

In 1995, Chinese proposed to build a NEO Search Telescope to take part in the international NEO joint survey at the International Conference on Near Earth Objects held at the United Nations, New York and at the Planetary Defense Workshop held in Livermore, California. After a long period of preparation from 1999, the telescope and site construction were carried out at Xuyu Station of the PMO. 


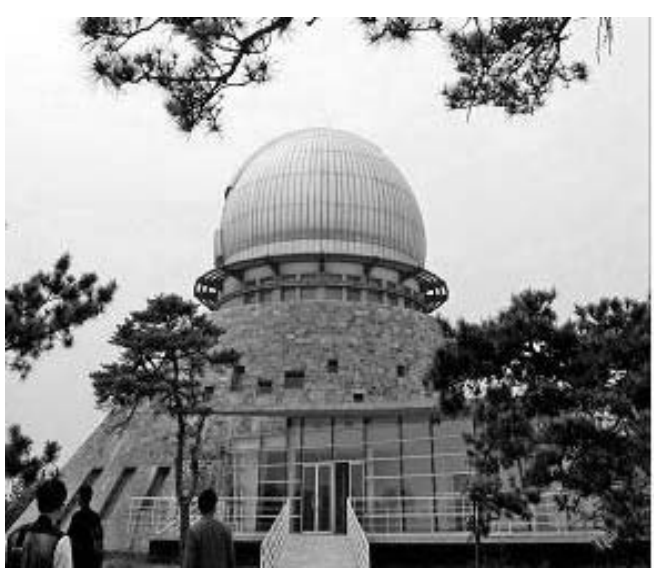

Figure 1. The dome for the telescope

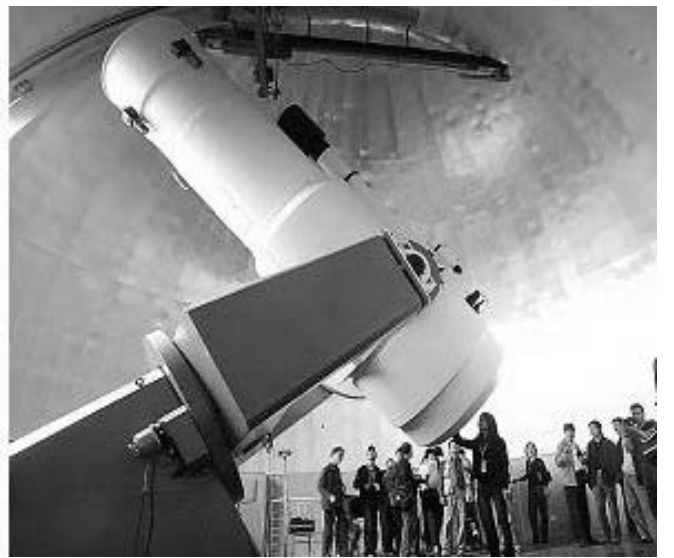

The 1.0/1.2 $m$ Schmidt Telescope

\section{1.0/1.2 $m$ Schmidt telescope}

Figure 1 shows the main building for the telescope and the telescope mounted in the dome. The telescope is equipped with a $4096 \times 4096$ high quantum efficiency CCD camera (Figure 2) with a field of view (FoV) of $2^{\circ} \times 2^{\circ}$. It will soon start operations after the last course to test the optical system and mechanical and electrical controls. The technical features of the instrument and the good quality of the sky at the site supply optimal conditions for an effective NEO survey. The high quantum efficiency of the CCD allows achieving deep magnitudes with short exposure times, while the wide FoV of the Schmidt telescope makes this facility competitive for the detection of new objects. The main purposes of the telescope are to look for NEOs and to carry out a NEO follow-up and recovery program according to the suggestions provided by the Minor Planet Center (MPC) and Spaceguard Central Node (SCN). After that, we will assess the impact probability of NEOs with Earth and establish a pre-alarm system for the Near Earth environment.

\subsection{Scientific background and objects}

The interest to study NEOs has grown steadily within the astronomical community on the grounds of science and the potential hazard to mankind. Recent studies (Bottke, Morbidelli, Jedicke, et al. 2002; D'Abramo, Harris, Boattini, et al. 2001) estimate the population of NEOs to be 900 members with $\mathrm{H}<18$ (corresponding to a diameter of about $1 \mathrm{~km}$ ). The estimated number of the objects with diameters larger than $100 \mathrm{~m}$ varies between 30,000 and 300,000.

At the end of 2005, 748 Potentially Hazardous Objects (PHOs) have been discovered, which is still far from the so-called Spaceguard goal, consisting of the discovery of $90 \%$ NEOs with $\mathrm{H}<18$ (more exactly, with $\mathrm{D}>1 \mathrm{~km}$ ). In fact, even though we estimate to be around halfway in the number of objects discovered to this size, all simulations show that the discovery of the remaining part to this size will require a longer time than the one that has been necessary to reach the present stage. Among the NEO groups, Atens are rather difficult to discover (Boattini \& Carusi 1997). This is due mostly to geometrical reasons; a large fraction of their orbits are inside that of the Earth, so most of the time these objects are observable only at small solar elongations. On the other hand, the main NEO surveys cover these regions of the sky only occasionally. Numerical simulations predict the existence of Inner Earth Objects (IEOs), asteroids or comets with orbits completely inside that of the Earth. But few of them have been discovered so far. 
This is not surprising, since the observational requirements for IEOs are even more stringent than those for Atens, requiring observations carried out even closer to the sun (no more than 75-80 degrees of elongation). To discover a larger fraction of Atens, and to start discovering IEOs, the above constraints need to be implemented in the sky survey.

The scientific objectives of the telescope summarized explicitly are

(i) Survey NEOs,

(ii) Determine the orbits of asteroids and comets,

(iii) Predict possible collision events,

(iv) Research the dynamical evolution of the orbits of asteroids and comets,

(v) Inspect space debris.

\subsection{Main parameters and capability of the telescope}

The telescope is a 1.0/1.2 $\mathrm{m}$ Schmidt type with a $4 \mathrm{~K} \times 4 \mathrm{~K}$ drift scanning CCD camera. A special design allows fitting the whole camera inside the telescope tube, directly in the focal station of the Schmidt optical system. The camera is cooled by a CryoTiger PT30, which guarantees the camera working with the CCD chip stable at $-110{ }^{\circ} \mathrm{C}$ independently of the position of telescope. The CCD camera has the function of drift scanning so that the readout rate is clocked to the sidereal drift rate across the CCD. With $\mathrm{F}=1.8$ for the optical system of the Schmidt telescope, CCD camera delivers a $1.705 \mathrm{arcsec} / \mathrm{pixel}$ sampling that corresponds to a FoV about $2^{\circ} \times 2^{\circ}$. Its high quantum efficiency (up to $92 \%$ at the peak) and the extremely low dark current guaranteed by the low working temperature $\left(<0.007 e^{-} / \mathrm{pixel} / \mathrm{sec}\right.$ at $\left.-100{ }^{\circ} \mathrm{C}\right)$ with a full capacity of 100,000 electrons make this system very valuable on moving objects when very fast exposures are required to avoid the trailing loss problem. The CCD camera is a Lockheed CCD 486 operated with a multiport readout channel at $700 \mathrm{kHz}$ giving a readout time of 12 seconds. Read noise was about 6 electrons at $100 \mathrm{kHz}$ and 15 electrons at $400 \mathrm{kHz}$ under $-100^{\circ} \mathrm{C}$. The camera and controller are built by Spectral Instruments Inc. in Tucson, Arizona.

The telescope mechanical structure was built by Nanjing Astronomical Instrument Co. Ltd and PMO with new and more accurate encoders, better motors and an advanced control system. The telescope is now able to perform open loop tracking (without any guider) for periods of 1 minute within $1^{\prime \prime}$ stretching and to perform CCD guiding tracking for periods of 10 minutes within $1^{\prime \prime}$ stretching. Due to the Schmidt system, the telescope has a large $\mathrm{FoV}$ of $3.14^{\circ} \times 3.14^{\circ}$, and the center wavelength of the correcting lens is $656.3 \mathrm{~nm}$. The configuration of the hardware system is very suitable for our main scientific objective to search for NEOs. The detailed parameters are summarized as follows

(i) Diameter of the correcting lens: $104 \mathrm{~cm}$,

(ii) Diameter of the primary mirror: $120 \mathrm{~cm}$,

(iii) Focal ratio: $\mathrm{F}=\mathrm{f} / \mathrm{D}=1.8(\mathrm{f}=180 \mathrm{~cm})$,

(iv) Effective field: $3.14^{\circ}$ (linear diameter: $100 \mathrm{~mm}$ ),

(v) Limiting magnitude of the telescope: 20.7,

(vi) Center wavelength of the correcting Lens: $656.3 \mathrm{~nm}$,

(vii) Light power distribution: $80 \%$ of light in less than $2^{\prime \prime}$ (linear diameter is less than $20 \mu m)$,

(viii) Distortion caused by optical designing and machining: less than $15 \mu \mathrm{m}$,

(ix) Bearing of the tube: bending to focal plane less than $0.02 \mathrm{~mm}$,

(x) Tracking precision: $1^{\prime \prime} / 4$ min,

(xi) Pointing Precision: less than $10^{\prime \prime}$. 


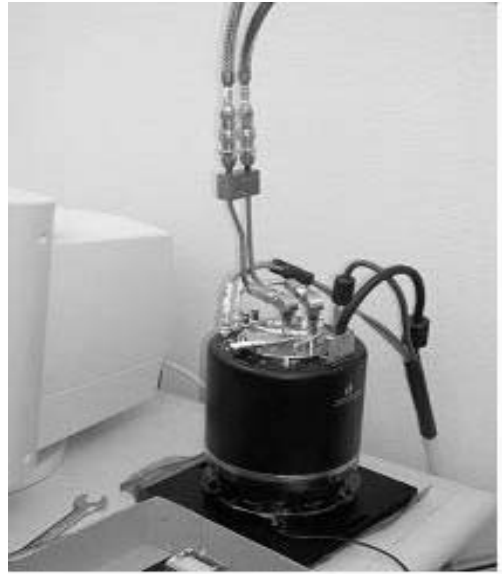

Figure 2. $4 \mathrm{~K}$ by $4 \mathrm{~K}$ CCD camera

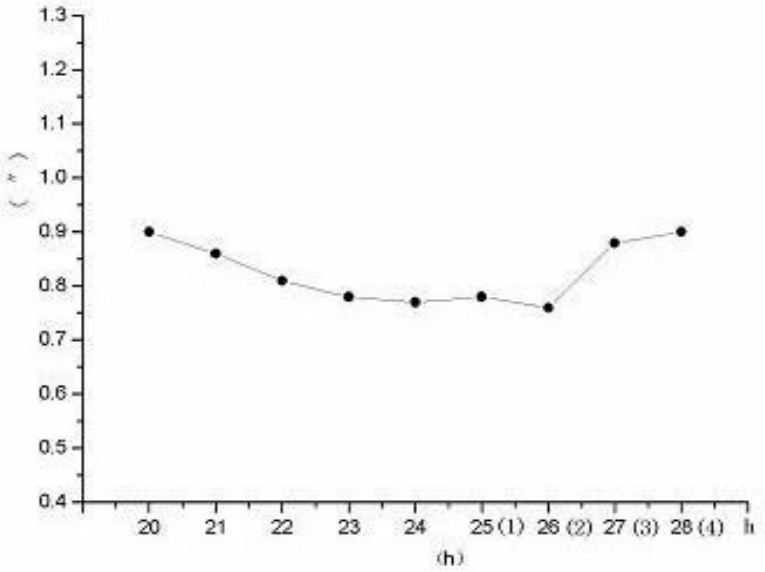

Figure 3. Seeing curve of the site

\section{Site of the observational station}

The NEO Search Telescope is mounted at Xuyu station. The site is $120 \mathrm{~km}$ north of Nanjing. Its position is at $N 32^{\circ} 44^{\prime} .2 \pm 0.5^{\prime}, E 118^{\circ} 27^{\prime} .9 \pm 0.5^{\prime}$ and the elevation above sea level is $180.9 \mathrm{~m}$. The survey will cover sky north of declination $S 20^{\circ}$. By historical record, the observing nights from 1995-1999 averaged 214.6 per year, and from 1990-1999 207.5 per year. We observed from Aug 1999 to July 2000 for a whole year and got 194 observing nights. In that year the number of astronomical observing hours was 1652 . Seeing Observation Results: From June to December in 1999, we made 1493 estimates of seeing; the average result is $\theta=0.81^{\prime \prime}$.

Figure 3 is the seeing curve and it shows that seeing changes symmetrically about midnight.

\section{Acknowledgements}

This work was supported by Natural Science Foundation of China (No. 10273024, 10573037, 10503013) and the Minor Planetary Foundation of Purple Mountain Observatory. The paper was finished at Tuorla Observatory, Piikkio, Finland during the exchange program between Finnish Academy and NSFC was performed. We thank the referee R.S. McMillan for useful comments and for taking care of language editing.

We would like to acknowledge Chinese Ministry Of Sciences and Technology (MOST), Chinese Academy of Sciences (CAS) and Jiangsu Province of China for the financial support for the telescope construction.

\section{References}

Boattini, A. \& Carusi, A. 1997, Vistas in Astronomy 41, 527

Bottke, W. F., Morbidelli, A., Jedicke, R., Petit, J.-M., Levison, H. F., Michel, P., Metcalfe, T. S. 2002, Icarus 156, 399

Carusi, A., Gehrels, T., Helin, E. F., Marsden, B. G., Russell, K. S., Shoemaker, C. S., Shoemaker, E. M., Steel, D. I. 1994, in: T. Gehrels (ed.), Hazards Due to Comets and Astroids (University of Arizona Press, Tucson), p. 127

D'Abramo, G., Harris, A. W., Boattini, A., Werner, S. C., Harris, A. W., Valsecchi, G. B. 2001, Icarus 153,214

Gehrels, T. \& Jedicke, R. 1995, Earth, Moon and Planets 72, 233

Stokes, G. H., Evans, J. B., Viggh, E. M., Shelly, F. C., \& Pearce, E. C. 2000, Icarus 148, 21 